\title{
A Combination of Drug-Eluting Stents and Bioresorbable Vascular Scaffolds in the Treatment of Multivessel Coronary Artery Disease
}

*Ahmed Al-Mamary, Filippo Zilio, Massimo Napodano

$$
\text { معالجة امراض الشرايين التاجية متعددة الأوعية باستخدام توليفة من الدعامات الدوائية الحلديثة }
$$

أحمد المعمرى، فيليبو زيليو، ماسيمو نابودانو

\begin{abstract}
Optimal management of multivessel coronary artery disease can be complex. We report a 67-year-old male patient who was admitted to the Padua University Hospital, Padua, Italy, in 2014 with a non-ST-elevation myocardial infarction. Coronary angiography showed diffuse multiple sub-occlusive lesions of the proximal and distal left coronary vessels involving a long segment of the vessel. On intravascular ultrasonography (IVUS), the left main artery was moderately diseased with critically stenotic and calcified branch ostia. A successful percutaneous coronary intervention using the T-stenting and small protrusion technique with two drug-eluting stents (DES) was performed on the left main artery and its main branches. Two bioresorbable vascular scaffolds were also deployed in overlap at the mid to distal segments of the left anterior descending artery and overlapping a previous DES at the proximal segment. The full expansion and apposition of the struts and scaffolds to the vessel wall without residual stenosis was confirmed by IVUS.
\end{abstract}

Keywords: Drug-Eluting Stents; Percutaneous Coronary Intervention; Ultrasonography; Coronary Stenosis; Myocardial Infarction; Case Report; Italy.

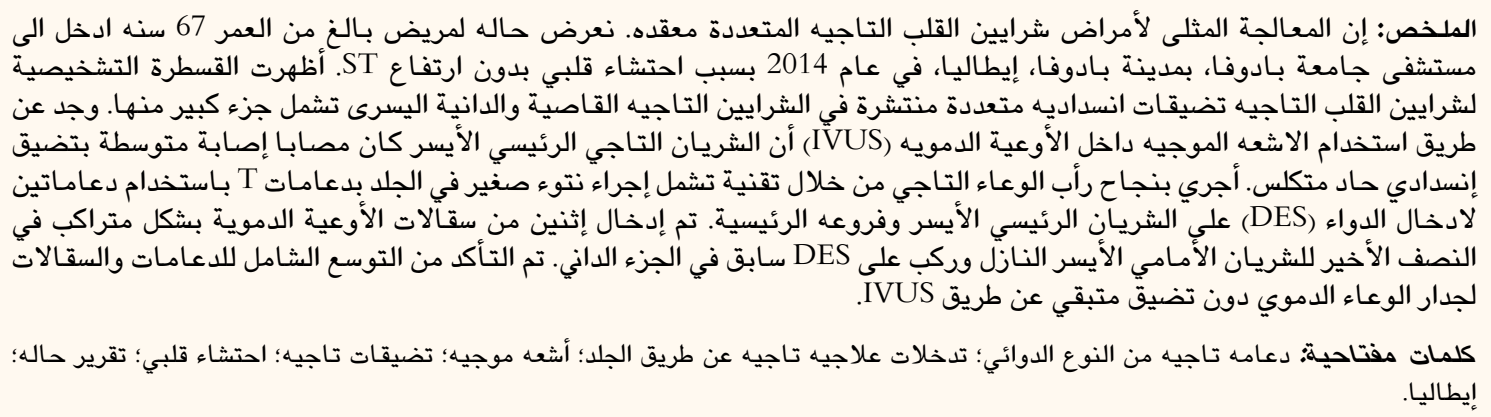

\section{$\mathrm{T}$} He optimal management of Multivessel coronary artery disease (CAD) is a complex medical decision with significant prognostic implications. Despite the advent of clinical and angiographic scores to aid treatment delineation, therapy for multivessel CAD should be tailored in each case with regards to the patient's clinical presentation. ${ }^{1}$ Coronary artery bypass grafting (CABG) surgery and percutaneous coronary intervention (PCI) are potential treatment options for patients with advanced CAD evaluated using the SYNTAX score, as stated in the European Society of Cardiology and the European Association for Cardio-Thoracic Surgery guidelines on myocardial revascularisation. ${ }^{2}$ This case describes a patient with multivessel CAD who was treated successfully with a combination of drug-eluting stents and bioresorbable vascular scaffolds.

\section{Case Report}

A 67-year-old male was admitted to the Department of Cardiac, Thoracic \& Vascular Sciences, Padua University Hospital, Padua, Italy, in 2014 with a non-ST-elevation myocardial infarction. He had $\mathrm{T}$ wave inversion in leads V1-4 and mildly increased myocardial necrosis biomarkers (peak troponin I: $2.770 \mu \mathrm{g} / \mathrm{L}$; upper reference value: $0.015 \mu \mathrm{g} / \mathrm{L}$ ). The patient had previously experienced a silent myocardial 

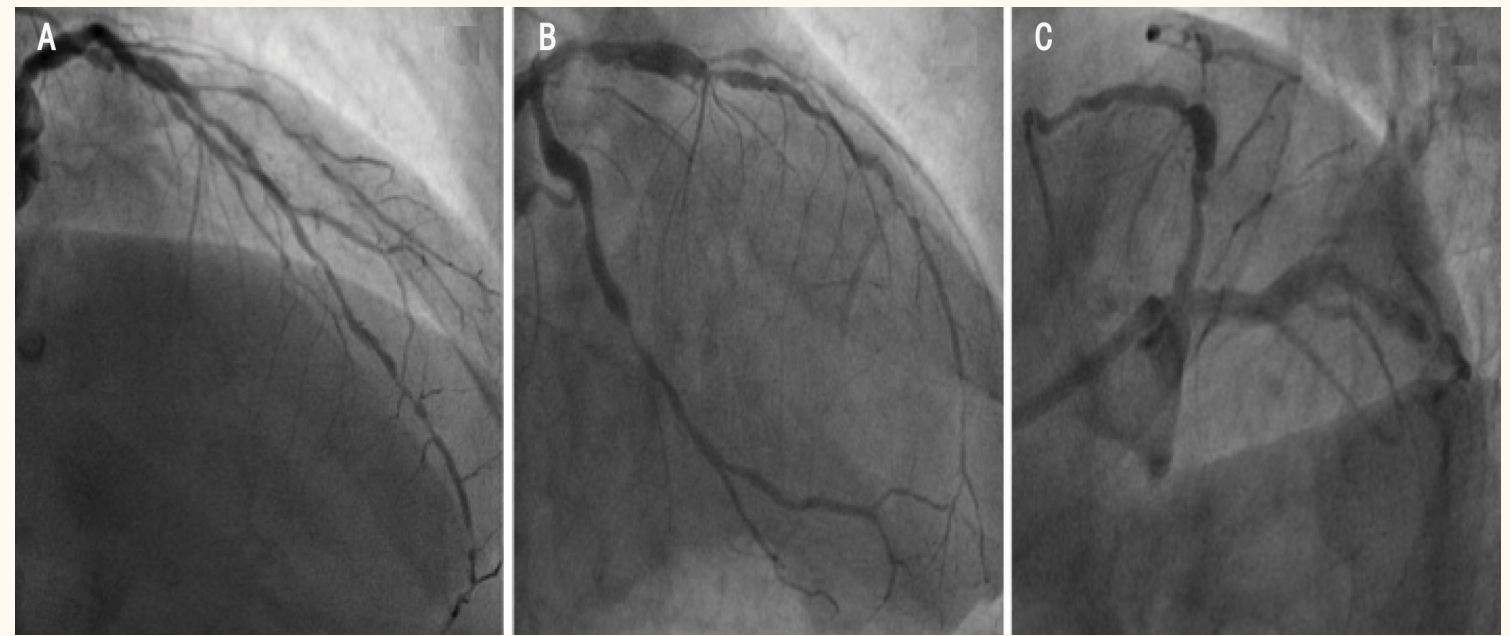

Figure 1A-C: Coronary angiography of a 67-year-old male patient from the (A) cranial right anterior oblique (RAO), (B) caudal RAO and (C) caudal left anterior oblique views showing multiple diffuse critical lesions of the proximal and distal segments of the left coronary arteries involving the left main trunk with a hazy proximal left circumflex artery (LCx) lesion. Despite evaluation from several planes, the stenosis of the proximal LCx artery remained unclear.

infarction without significant clinical sequelae as confirmed by echocardiographic follow-up which showed normal dimensions with preserved systolic left ventricular function (left ventricular end-diastolic volume index: $65 \mathrm{~mL} / \mathrm{m}^{2}$; ejection fraction: $58 \%$ ) without wall motion abnormalities. At this time, the patient was asymptomatic without residual ischaemia. This was confirmed by a negative exercise stress test at $150 \mathrm{~W}$. The patient was a smoker and had several other cardiovascular risk factors, including hypertension, dyslipidaemia and type 2 diabetes mellitus.

At presentation, the patient was immediately administered $250 \mathrm{mg}$ of intravenous aspirin and $180 \mathrm{mg}$ of oral ticagrelor. As he had a high Global Registry of Acute Coronary Events risk score (145), an emergency coronary angiogram was carried out using the right radial artery. This revealed non-significant stenosis of the dominant right coronary artery and diffuse calcified and atherosclerotic segments with multiple sub-occlusive proximal and distal left CAD lesions involving the left main trunk with haziness at the proximal segment of the left circumflex (LCx) artery. Despite evaluation from several planes, the angiographic assessment was inconclusive and the stenosis of the proximal LCx artery remained unclear [Figure 1]. Intravascular ultrasonography (IVUS) using a catheter (Eagle Eye ${ }^{\circledR}$ Catheter, Volcano Corp., San Diego, California, USA) revealed that the left main trunk was moderately diseased [Figure 2] with critically calcified branch ostia. The angiographic SYNTAX score of the patient was calculated to be 40 .

Due to the anatomical complexity of the distal coronary arteries, the patient was referred for a multiple lesion PCI. A decision was made to combine rigid cage stents for the proximal wide lumen segments and bioresorbable vascular scaffolds for the diffuse
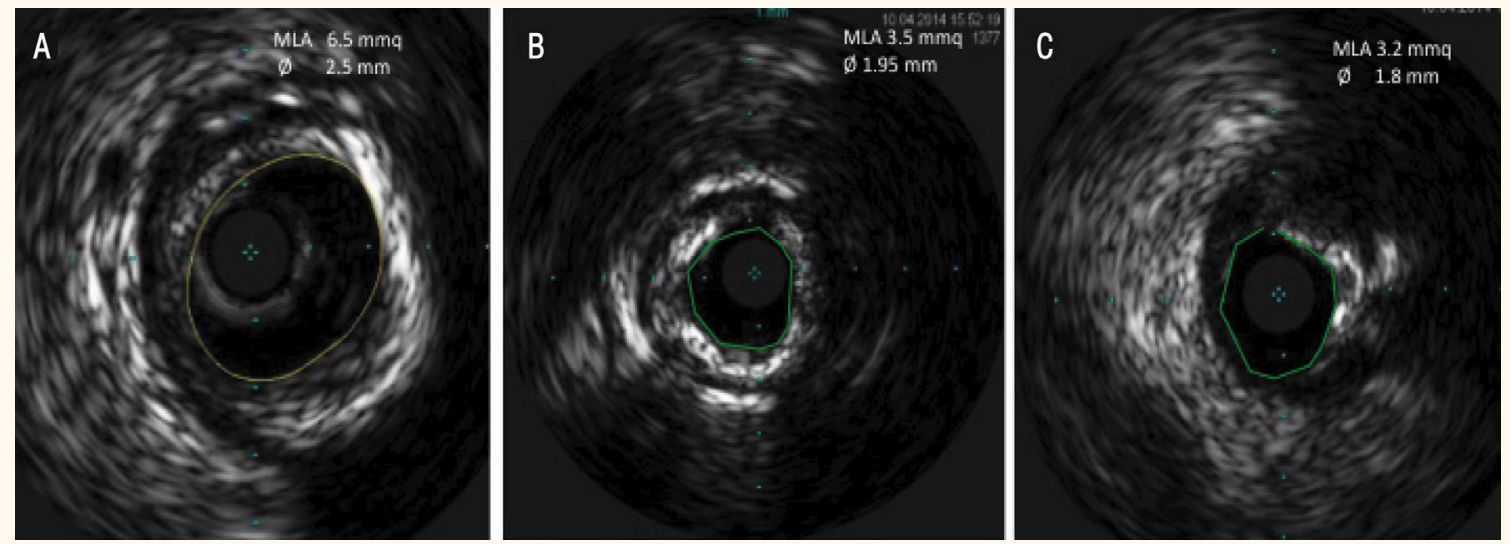

Figure 2A-C: Intravascular ultrasonography (IVUS) of a 67-year-old male patient showing (A) moderate left main trunk disease, (B) multiple significant stenoses of the left anterior descending artery and (C) stenosis of the proximal left circumflex artery with eccentric superficial and deep dense calcific plaque (arrow).

$M L A=$ minimal lumen area . 

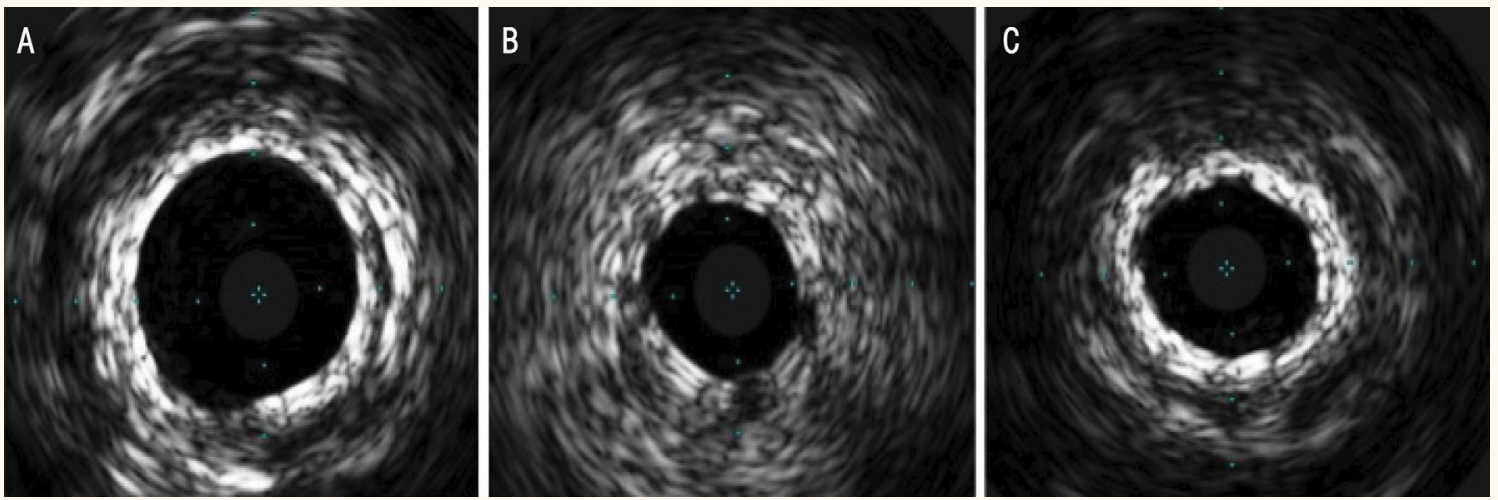

Figure 3A-C: Post-interventional cross-sectional intravascular ultrasonography of a 67-year-old male patient following the implantation of two bioresorbable vascular scaffolds and two drug-eluting stents. The stents were well-apposed and expanded with no signs of dissection at the (A) left main trunk, (B) proximal portion of the left anterior descending artery and $(\mathbf{C})$ proximal portion of the left circumflex artery.

atherosclerotic lesions which involved the long middistal segments of the left anterior descending (LAD) artery. Due to severe calcifications on the mid-distal LCx artery, percutaneous transluminal coronary angioplasty was performed. Instead of a bioresorbable vascular scaffold, a single $2.5 \times 23.0 \mathrm{~mm}$ everolimuseluting stent (Xience Prime ${ }^{\circledR}$, Abbott Vascular, Santa Clara, California, USA) was implanted at the middistal LCx artery in order to avoid suboptimal scaffold expansion/apposition. After preparation of the lesions with semi-compliant balloons, a successful two-stent approach using the T-stenting and small protrusion technique was performed at the left main trunk and its main branches using a $3.5 \times 33.0 \mathrm{~mm}$ everolimus-

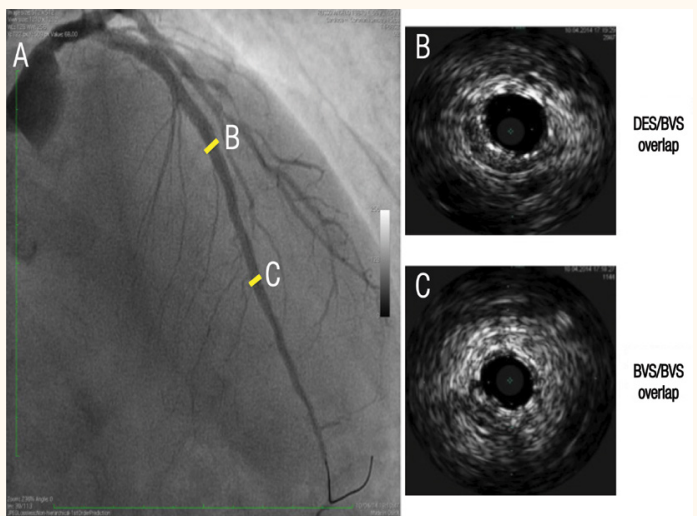

Figure 4A-C: A: Post-interventional coronary angiography of a 67 -year-old male patient following the implantation of two bioresorbable vascular scaffolds at the mid-distal portion of the left anterior descending artery and in overlap with a drug-eluting stent at the proximal portion. B and C: Cross-sectional intravascular ultrasonography images corresponding to sections of the coronary angiogram (yellow lines) showing the uniform and full expansion of the struts and scaffolds apposed to the intimo-medial membrane. All three concentric stents and scaffold layers were noted at overlap sites as discrete echo-bright structures. $D E S=$ drug-eluting stent; $B V S=$ bioresorbable vascular scaffold. eluting stent (Xience Prime ${ }^{\circledR}$ ) at the level of the left main trunk-proximal segment of the LAD artery. This was followed by another $3.5 \times 12.0 \mathrm{~mm}$ everolimus-eluting stent (Xience Prime ${ }^{\circledR}$ ) at the left main trunk-proximal segment of the LCx artery. Kissing balloon postdilation was then performed in the bifurcation of the left main, LAD and LCx coronary arteries. Finally, after the mid-distal LAD artery lesions were prepared using an incremental semi-compliant balloon, excluding the recurrent apical segment, two bioresorbable vascular scaffolds (Absorb Bioresorbable Vascular Scaffold, Abbott Vascular) of $3.0 \times 28 \mathrm{~mm}$ and $2.5 \times 28 \mathrm{~mm}$, respectively, were positioned proximally to the distal segments, with minimal overlap (marker-to-marker implantation) at the mid- to mid-distal segment of the LAD artery and overlapping the metallic cage stent at the proximal segment of the LAD artery.

Following the intervention, a coronary angiogram showed that all vessel side branches were maintained without evidence of any complications. Full expansion and complete apposition of the metallic struts to the vessel wall at the proximal segments was confirmed via IVUS [Figure 3]. Similarly, the uniform and full expansion of the bioresorbable scaffolds apposed to the intimo-medial membrane at the mid-distal LAD artery was observed without residual stenosis via angiography and IVUS [Figure 4]. The patient remained asymptomatic without further cardiac events and was discharged one week after admission on a 12-month course of dual antiplatelet therapy. At a six month clinical follow-up, the patient was symptom-free.

\section{Discussion}

Consideration of the coronary anatomy, disease complexity, the presence of left ventricular dysfunction and SYNTAX risk score, along with any 
comorbidities, interventional risks, long-term outcomes, local expertise and the patient's personal preferences, are imperative when making decisions regarding treatment for patients with multivessel CAD. ${ }^{1,3}$ For patients with SYNTAX scores over 22, CABG surgery is associated with better long-term outcomes than PCI, barring the presence of excessive preoperative risk factors. ${ }^{3}$ Research indicates that diabetic patients affected by $\mathrm{CAD}$ respond better to CABG treatment over PCI, particularly patients with severe and diffuse CAD. ${ }^{4-6}$ One recent trial involving patients with diabetes mellitus and advanced multivessel CAD found that CABG treatment was beneficial in that it significantly reduced rates of death, myocardial infarction and repeated revascularisation at a five-year follow-up, although a higher rate of strokes was observed. ${ }^{6}$ Based on this trial, CABG surgery is recommended as the preferred treatment for patients with multivessel CAD, as it has the most favourable long-term outcomes in patients with a high SYNTAX score but without high surgical risk. ${ }^{4,6}$ Alternatively, patients who are at high risk for surgery, or who have low-severity CAD, should undergo PCI treatment. ${ }^{5}$ The patient in the current case presented with a high-risk SYNTAX score and angiographic evidence of multivessel CAD; treatment with PCI was chosen as CABG surgery was deemed unsuitable due to the anatomical complexity of the distal coronary arteries. As distal coronary stenoses may affect adenosine-mediated vasodilation leading to a falsenegative result, the fractional flow reserve technique was not used to evaluate the PCI target.

The present case illustrates the importance of tomographic techniques-such as IVUS imagingover coronary angiography in the diagnosis and management of complex multivessel CAD. In the current case, IVUS showed moderate left main trunk disease and critically calcified ostial lesions of the LAD and LCx arteries. However, in comparison with conventional intravascular imaging modalities such as IVUS, optical coherence tomography has a higher resolution (range: 10-15 $\mu \mathrm{m}$ ), allowing for the more accurate detection and quantification of scaffold malapposition, underexpansion, tissue prolapse or stent edge dissection. Recently, research has shown that optical coherence tomography can be used to identify adverse cardiac features requiring immediate intervention in a large proportion of patients, resulting in improved clinical outcomes. ${ }^{7}$ Unfortunately, this alternative imaging technology was not available at the Padua University Hospital at the time of the intervention.

The implantation of multiple metallic stents covering long coronary segments can substantially affect vasomotion and may cause vascular inflammation, restenosis, thrombosis and neoatherosclerosis. $^{8-10}$ Permanent metallic platform stents also indefinitely impair the physiological vasomotor functioning of the vessel; hence the coronary geometry and systo-diastolic movement of coronary arteries treated with metallic stents remain similar up to a year after implantation. ${ }^{9,10}$ Bioresorbable vascular scaffolds have the potential to overcome these limitations as they provide a temporary scaffolding which disappears after 2-3 years, liberating the treated vessel, and can help to restore the vessel's original shape, preserving laminar blood flow, reducing the plaque-to-media ratio and restoring the vasomotion, pulsatility, cyclical strain, adaptive shear stress and mechanotransduction of the vessel. ${ }^{8,9,11}$ Following PCI, the absence of any residual foreign material and restoration of functional endothelial coverage can also reduce the risk of stent thrombosis, myocardial infarction, sudden death and the need for long-term dual antiplatelet therapy; in addition, PCI does not exclude the possibility of future graft anastomosis in case the need for future bypass surgery arises. ${ }^{8,9}$ However, there are as yet no data with regards to the feasibility of performing CABG surgery at coronary sites which have previously undergone implantation of a bioresorbable vascular scaffold.

After implantation, the bioresorbable vascular scaffold maintains sufficient radial strength to prevent vessel recoil and releases the drug to inhibit the proliferation of neointima. However, the scaffold's mass and radial strength gradually decrease over time and it vanishes completely after 2-3 years, leaving the vessel covered with a healthy endothelium and with normal vasomotor function. ${ }^{8}$ Bioresorbable vascular scaffolds should be predominantly used in young patients for whom multiple long stents are required, particularly those with long lesions or diffuse disease; patients with lesions located in the mid-distal portion of the coronary arteries will also benefit because the use of bioresorbable vascular scaffolds does not preclude potential bypass grafting in the future. ${ }^{8}$ For severely calcified vessels, preparation of the lesion with optimal pre-dilatation or the use of additional devices and procedures such as cutting and scoring balloons or rotational atherectomy is crucial for the successful implantation of the scaffold. Even severely calcified vessels will benefit from the disappearance of the scaffold, as this reduces the risk of late stent thrombosis and can lead to late lumen gain. ${ }^{8}$

No guidelines currently exist on the simultaneous use of drug-eluting stents and bioresorbable vascular scaffolds to manage diffuse critical atherosclerotic multivessel CAD. Gil et al. recently reported that the 
concurrent use of these devices might be safe and effective, with no mortality or incidents of myocardial infarction or stent thrombosis observed at a 12 month follow-up and very few cases of clinically-driven target lesion revascularisation. ${ }^{12}$ The rationale for using both devices in the current case was to treat the diffuse disease percutaneously while avoiding the use of long metallic stented segments. Due to the diffuse disease at the mid-distal LAD artery, the patient's anatomy was not deemed suitable for CABG surgery. On the other hand, the presence of lesions at the left main trunk and proximal LAD artery supports coronary revascularisation over medical treatment, especially for acute coronary syndromes such as nonST-elevation myocardial infarctions., ${ }^{2,13}$ As a result, PCI was performed; however, as multiple lesions and segments required treatment, coronary bioresorbable vascular scaffolds were used to avoid mid-to-longterm drug-eluting stent-related adverse events (e.g. stent thrombosis and restenosis). ${ }^{8,9}$ In addition, implanting a long metallic drug-eluting stent at the distal coronary bed could potentially affect coronary flow or the outcome of future CABG surgeries. ${ }^{9,10}$ Nevertheless, metallic stents were also implanted at proximal sites due to the limitations of bioresorbable vascular scaffolds for large vessels, bifurcations (i.e. the left main trunk lesion in the current patient) and severely calcified lesions., ${ }^{9,11}$

\section{Conclusion}

Lesions in the left main coronary artery are rarely isolated and the coronary anatomy is not always suitable for a CABG surgical approach, regardless of risk scores. As with the current case, a combination of drug-eluting stents and bioresorbable vascular scaffolds can be a feasible treatment option for complex diffuse multivessel CAD with left main trunk involvement. The use of non-metallic bioresorbable vascular scaffolds in long diffuse lesions of the middistal vessels, in combination with metallic struts in the proximal segment, may mitigate the adverse effects of metallic stents. This technique ensures adequate and temporary scaffolding while also promoting adequate healing of the vessel wall and allowing for the possibility of future graft anastomosis should the patient subsequently need CABG surgery.

\section{References}

1. Parikh PB, Kirtane AJ, Moses JW. Management of multivessel coronary artery disease. Panminerva Med 2013; 55:311-26.

2. Kolh P, Windecker S, Alfonso F, Collet JP, Cremer J, Falk V, et al. 2014 ESC/EACTS guidelines on myocardial revascularization: The Task Force on Myocardial Revascularization of the European Society of Cardiology (ESC) and the European Association for Cardio-Thoracic Surgery (EACTS) - Developed with the special contribution of the European Association of Percutaneous Cardiovascular Interventions (EAPCI). Eur J Cardiothorac Surg 2014; 46:517-92. doi: 10.1093/ejcts/ezu366.

3. Deb S, Wijeysundera HC, Ko DT, Tsubota H, Hill S, Fremes SE. Coronary artery bypass graft surgery vs percutaneous interventions in coronary revascularization: A systematic review. JAMA 2013; 310:2085-95. doi: 10.1001/ jama.2013.281718.

4. Dangas GD, Farkouh ME, Sleeper LA, Yang M, Schoos MM, Macaya $C$, et al. Long-term outcome of PCI versus CABG in insulin and non-insulin-treated diabetic patients: Results from the FREEDOM trial. J Am Coll Cardiol 2014; 64:1189-97. doi: 10.1016/j.jacc.2014.06.1182

5. Nicolini F, Agostinelli A, Vezzani A, Benassi F, Gherli T. CABG versus $\mathrm{PCI}$ in the treatment of diabetic patients affected by coronary artery disease: Which is the best option? Int Heart J 2014; 55:469-73. doi: 10.1536/ihj.14-061.

6. Farkouh ME, Domanski M, Sleeper L, Siami FS, Dangas G, Mack $M$, et al. Strategies for multivessel revascularization in patients with diabetes. N Engl J Med 2012; 367:2375-84. doi: 10.1056/NEJMoa1211585.

7. Allahwala UK, Cockburn JA, Shaw E, Figtree GA, Hansen PS, Bhindi R. Clinical utility of optical coherence tomography (OCT) in the optimisation of Absorb bioresorbable vascular scaffold deployment during percutaneous coronary intervention. EuroIntervention 2015; 10:1154-9. doi: 10.4244/ EIJV10I10A190.

8. Lesiak M, Araszkiewicz A. "Leaving nothing behind": Is the bioresorbable vascular scaffold a new hope for patients with coronary artery disease? Postepy Kardiol Interwencyjnej 2014; 10:283-8. doi: 10.5114/pwki.2014.46940

9. Iqbal J, Onuma Y, Ormiston J, Abizaid A, Waksman R, Serruys P. Bioresorbable scaffolds: Rationale, current status, challenges, and future. Eur Heart J 2014; 35:765-76. doi: 10.1093/eurheartj/ eht542.

10. Gomez-Lara J, Brugaletta S, Farooq V, van Geuns RJ, De BruyneB, Windecker S, et al. Angiographic geometric changes of the lumen arterial wall after bioresorbable vascular scaffolds and metallic platform stents at 1 -year follow-up. JACC Cardiovasc Interv 2011; 4:789-99. doi: 10.1016/j.jcin.2011.04.009.

11. Tamburino C, Latib A, van Geuns RJ, Sabate M, Mehilli J, Gori T, et al. Contemporary practice and technical aspects in coronary intervention with bioresorbable scaffolds: A European perspective. EuroIntervention 2015; 11:45-52. doi: 10.4244/ EIJY15M01_05.

12. Gil RJ, Bil J, Pawłowski T, Yuldashev N, Kołakowski L, Jańczak J, et al. The use of bioresorbable vascular scaffold Absorb BVS ${ }^{\circledR}$ in patients with stable coronary artery disease: One-year results with special focus on the hybrid bioresorbable vascular scaffolds and drug eluting stents treatment. Kardiol Pol 2016; 74:627-33. doi: 10.5603/KP.a2015.0250.

13. Hahalis G, Alexopoulos D. Revascularization strategies in multivessel and left main coronary artery disease: SYNTAX and beyond. Hellenic J Cardiol 2014; 55:328-35. 